\title{
Video-guided calibration of an augmented reality mobile $\mathrm{C}$-arm
}

\author{
Xin Chen • Hemal Naik • Lejing Wang • \\ Nassir Navab • Pascal Fallavollita
}

\begin{abstract}
Purpose The augmented reality (AR) fluoroscope augments an X-ray image by video and provides the surgeon with a real-time in situ overlay of the anatomy. The overlay alignment is crucial for diagnostic and intra-operative guidance, so precise calibration of the AR fluoroscope is required. The first and most complex step of the calibration procedure is the determination of the X-ray source position. Currently, this is achieved using a biplane phantom with movable metallic rings on its top layer and fixed X-ray opaque markers on its bottom layer. The metallic rings must be moved to positions where at least two pairs of rings and markers are isocentric in the X-ray image. The current "trial and error" calibration process currently requires acquisition of many X-ray images, a task that is both time consuming and radiation intensive. An improved process was developed and tested for C-arm calibration.

Methods Video guidance was used to drive the calibration procedure to minimize both X-ray exposure and the
\end{abstract}

X. Chen $\cdot$ H. Naik $\cdot$ L. Wang $\cdot$ N. Navab $\cdot$ P. Fallavollita $(\bowtie)$

Chair for Computer Aided Medical Procedures, Fakultät für Informatik, Technische Universität München, Munich, Germany

e-mail: fallavol@in.tum.de

X. Chen

e-mail: chenxin@in.tum.de

H. Naik

e-mail: hmnaiks@gmail.com

L. Wang

e-mail: wangle@in.tum.de

N. Navab

e-mail: navab@cs.tum.edu time involved. For this, a homography between X-ray and video images is estimated. This homography is valid for the plane at which the metallic rings are positioned and is employed to guide the calibration procedure. Eight users having varying calibration experience (i.e., 2 experts, 2 semi-experts, 4 novices) were asked to participate in the evaluation.

Results The video-guided technique reduced the number of intra-operative X-ray calibration images by $89 \%$ and decreased the total time required by $59 \%$.

Conclusion A video-based C-arm calibration method has been developed that improves the usability of the AR fluoroscope with a friendlier interface, reduced calibration time and clinically acceptable radiation doses.

Keywords C-arm fluoroscopy · Medical augmented reality · Multi modalities · Visualization · Video guidance · Calibration · Orthopedic and trauma surgery

\section{Introduction}

Orthopedic and trauma interventions heavily rely on intraoperative X-ray images to visualize bone configuration and guide treatments, especially in minimally invasive surgery. Within the last two decades, mobile $\mathrm{C}$-arm fluoroscopes have become the everyday tool for surgeons to navigate surgery. However, surgeons can only visualize the X-ray images on a monitor inside the operating room. Therefore, it becomes essential for the surgeon to mentally map X-ray information to the operation site in order to build a spatial relation between the bone anatomy, implants, surgical instruments, and patient. This introduces additional difficulties and can compromise the outcome quality of surgeries. 


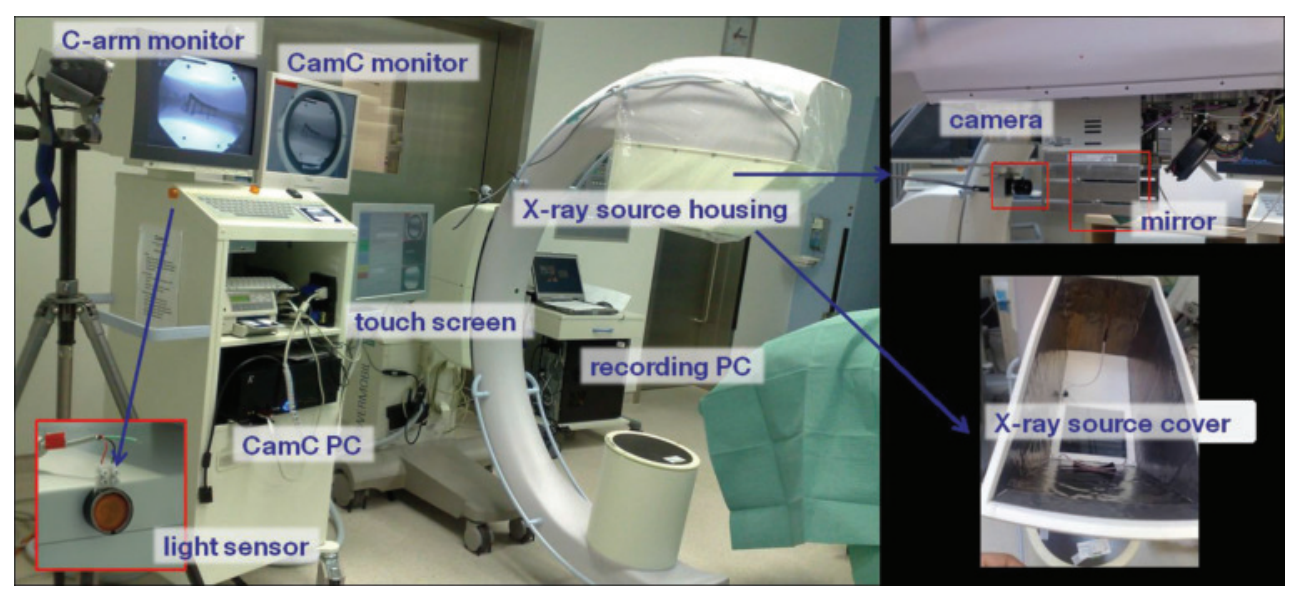

Fig. 1 The augmented reality fluoroscope is conceived by a camera and mirror construction attached to the X-ray source housing of a mobile C-arm device

\section{Prior art of IGS and AR technologies}

To maximize successful patient outcomes, image-guided surgery (IGS) has been developed offering surgeons improved visual perception and guidance during surgery. IGS augments and complements the physician's ability to clearly and quickly perceive the different structures of anatomy and surgical instruments. It is achieved by fusing relevant information in a common coordinate system based on registration and tracking techniques. Today, medical augmented reality (AR) systems exist, which enhance the direct view of the surgical target with virtual data. Exemplary setups and applications for in situ visualization include augmented reality operating microscopes for neurosurgery [1,2], head-mounted operating binoculars for maxillofacial surgery [3], augmentation of MRI data onto an external camera view for neurosurgery [4], and systems based on head-mounted displays [5-8]. A system based on a tracked semi-transparent display for in situ augmentation has also been proposed [9]. An extensive literature review on medical augmented reality can be found in [9].

Most of the existing in situ visualization systems augment the view of a surgeon or optical video cameras with preoperative data. However, preoperative data cannot always represent the latest anatomical information specifically during the course of surgery since patient and organ movements are observed. For this, intra-operative imaging has been recently considered for medical augmented reality, since the images can be updated in real time during surgery. Some examples follow. First, Stetten et al. [10] present a real-time tomographic reflection augmenting ultrasound images of the anatomy with its surface based on a half silvered mirror and a flat panel miniature monitor. Fichtinger et al. [11] propose an intra-operative CT medical AR system for visualizing CT slice onto the patient in situ based on a half transparent mirror and a monitor setup rigidly attached to a CT scanner. A similar technique was proposed for the in situ visualization of a single MRI slice [12]. Feuerstein et al. [13] augments laparoscope video images with intra-operative $3 \mathrm{D}$ cone-beam CT by tracking a C-arm and laparoscope using the same external optical tracking system. Wendler et al. [14] fuses realtime ultrasound images with synchronized real-time functional nuclear information from a gamma probe by optical tracking. Other types of tracking exist and provide the realtime C-arm fluoroscopy pose via radiographic fiducials and intensity-based registration $[15,16]$. However, these systems require external tracking hardware and algorithms, hindered by line of sight issues, which require a specific and complex arrangement of the imaging devices in order to align the different images or viewing geometries.

\section{AR fluoroscopy}

The camera augmented mobile C-arm is composed of a mobile $\mathrm{C}$-arm enhanced by a camera and mirror unit $[17,18]$. Both X-ray source and video centers are virtually aligned at the same position so that both can have the same view of the patient (Fig. 1). From this, an intuitive in situ intra-operative overlay between X-ray and video images is achieved (Fig. 2). With the X-ray augmented by live video, surgeons can perform operations more confidently with less radiation exposure, reduced rate of potential surgical mistakes, and increased reproducibility.

The effective use of this augmented reality fluoroscope in different clinical applications has been demonstrated recently. These include: parallax-free $\mathrm{X}$-ray image stitching [19], visual servoing for the C-arm down-the-beam positioning [20], interlocking of intramedullary nailing [21-23] the six degree of freedom (DOF) kinematic modeling and inverse kinematics of a mobile C-arm fluoroscope [24], calibration of the AR fluoroscope [25,26], robust pose estimation of a Carm via AR markers [27], parallax-free panorama generation 

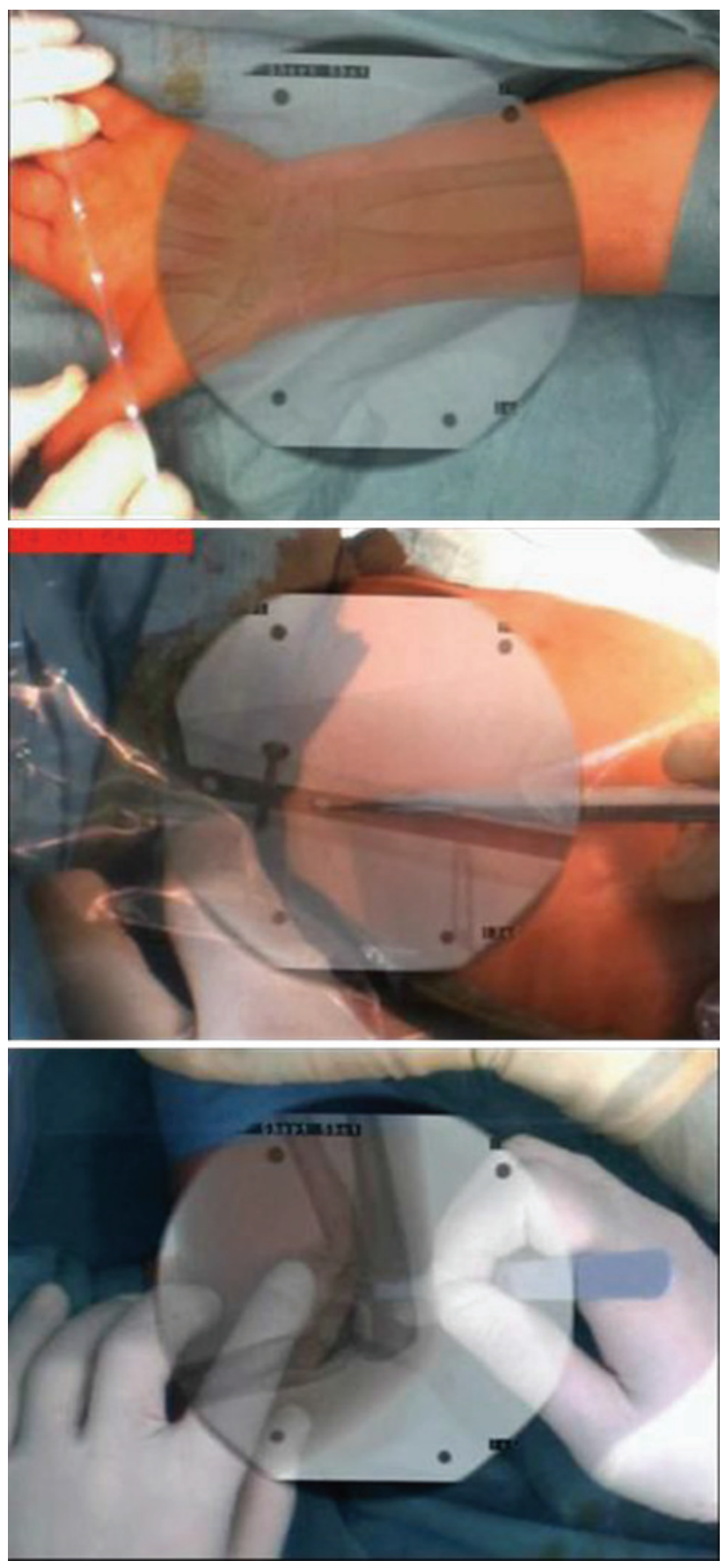

Fig. 2 In situ real-time intra-operative X-ray and optical images overlay provided by CamC. Top visualizing the structure of the distal radius, center defining the entry point on the skin on top of the implant locking hole, and bottom skin incision above an elbow fracture

for total knee arthroplasty surgery [28], and improved multimodal perceptual visualizations [29-31].

In its current form, the major drawback of the AR fluoroscopy device lies in its time-consuming and radiation intensive calibration procedure. This limitation becomes apparent if the system becomes mis-calibrated prior to or during surgery.

\section{Contributions}

An inexperienced user may require more than a dozen $\mathrm{X}$ ray image acquisitions and significant time to calibrate the AR fluoroscope [27]. Thus, the objective of this paper is to describe an elegant and simple solution, which would decrease the amount of radiation and time required to complete the calibration. In this light, a novel method is introduced such that users rely solely on video guidance to complete the task. To evaluate the feasibility of our approach, eight participants contributed in the experimentation. Finally, to assess the precision of our video-guided method the following metrics were measured: (i) the total number of X-ray images and (ii) the total amount of time required to complete the calibration.

\section{Existing calibration}

In this section, a brief description of the existing calibration framework is presented.

Framework

The basic idea behind the AR fluoroscopy system is that the alignment of X-ray source and optical camera centers can guarantee the overlay homography to be valid for all points in the field of view. In this manner, a real-time in situ image overlay between X-ray and video images is achieved. From (1), $\boldsymbol{H}$ is the overlay homography that can be calculated by: $\boldsymbol{K}_{\boldsymbol{v}}$ (calibration matrix of video camera), $\boldsymbol{K}_{\boldsymbol{x}}$ (calibration matrix of X-ray fluoroscope), $\boldsymbol{R}$ (rotation matrix from X-ray to video camera), $\boldsymbol{t}$ (translation vector from X-ray to video camera), $\boldsymbol{n}$ (normal vector of the plane where the points locate), and $\boldsymbol{d}$ (distance between the plane and X-ray fluoroscope center). The homography can then be used to register the image points in X-ray images onto video images. However, it is the translation vector $\boldsymbol{t}$ that makes the homography plane dependent, which means that the homography is only effective to the image points on the same plane. If $\boldsymbol{t}$ is not zero, each plane should have a homography of its own instead of one homography for the entire field of view. This makes the AR fluoroscope less reliable. As such, a bi-plane phantom (Fig. 3-bottom) was designed and a calibration process was proposed to minimize the translation vector and calculate the homography.

$H=K_{v} R K_{x}^{-1}+\frac{1}{d} K_{v} t n^{T} K_{x}^{-1}$

Five X-ray and video visible spherical markers are fixed on the bottom layer of the bi-plane phantom, and five movable metallic rings are put on the top layer. Using these five pairs of markers and rings, the alignment of X-ray source and 
Fig. 3 Top the estimation of a homography is required to align $\mathrm{X}$-ray and video centers. This is achieved using a Bi-plane calibration phantom (bottom) Through a series of "trial and image depicting the five pairs of iso-centric rings and markers needs to be acquired error" processes, an X-ray
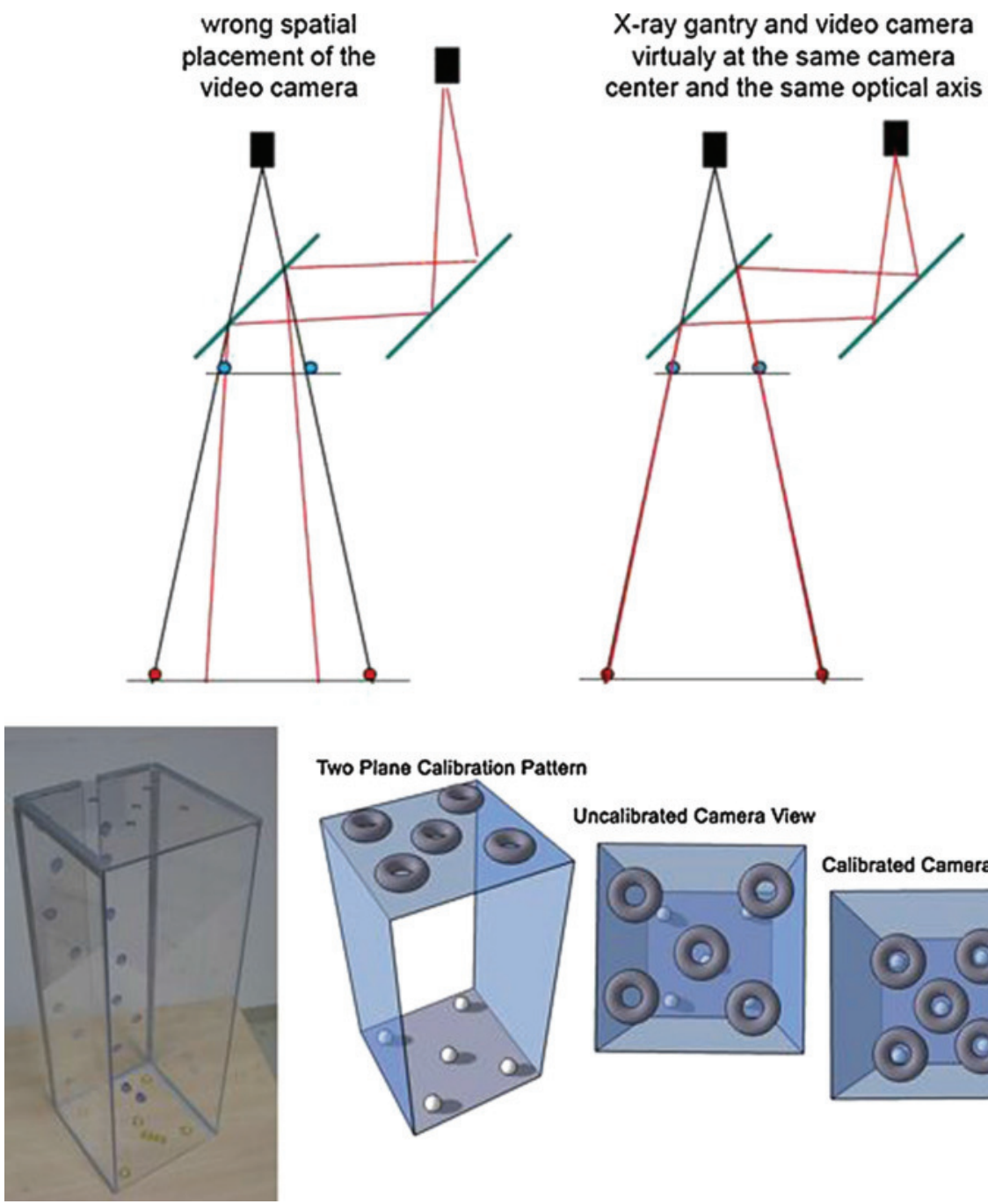

Two Plane Calibration Pattern

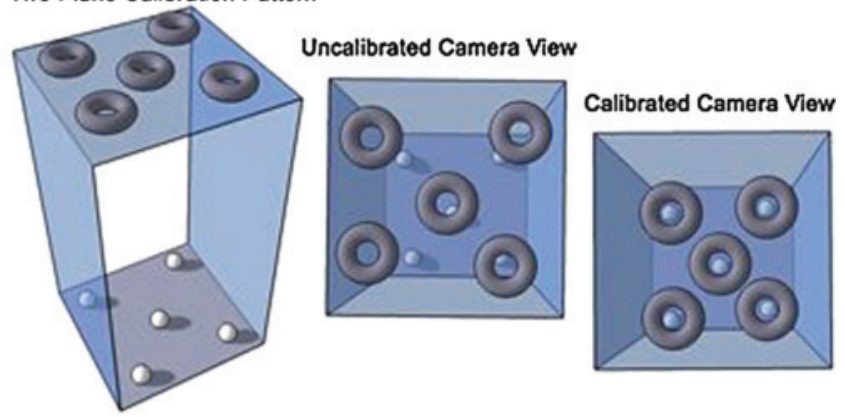

optical camera center is achieved. Readers are encouraged to view [25] for additional geometrical details characterizing the calibration.

\section{Alignment of X-ray source and video optical centers}

Having a geometrically correct overlay between the video and X-ray image requires both to be acquired from the exact same view point. The aim of the calibration is to position the AR fluoroscopy mirrors such that the optical camera center coincides with the X-ray source center (Fig. 3-top). The $\mathrm{X}$-ray source center is first determined by the intersection of at least two distinct linear rays, each of which is defined by two distinct points - the movable metallic ring on the top layer and the fixed X-ray visible marker on the bottom layer. The existing calibration method can only provide guidance based on X-ray images until all five metallic rings on the top layer are moved to the position where all five markers on the bottom layer can be seen through the five rings (isocentric), as shown in Fig. 3-bottom. This step needs many X-ray acquisitions, is time consuming, and is considered a "trial and error" process (i.e., move metallic rings, acquire X-ray, repeat, etc.). Then, in the second calibration phase, the mirrors are moved to align the two centers. The X-ray source and the optical camera center are aligned if and only if the five markers on the bottom layer can also be seen through five rings (iso-centric) in the video image. Moving the mirrors requires only a few seconds.

Homography estimation for overlay

After successful alignment of X-ray source and optical camera center, a universal non-plane-dependent homography $\boldsymbol{H}$ $\in R^{3 \times 3}$ can be calculated using either the calibration matrices of the X-ray fluoroscope and optical camera, together with rotation matrix between them, or points correspondence between X-ray and video images. However, the former method, which needs precise optical camera and X-ray fluoroscope calibration together with pose estimation, is complicated and error prone. Thus, the latter method is used and 


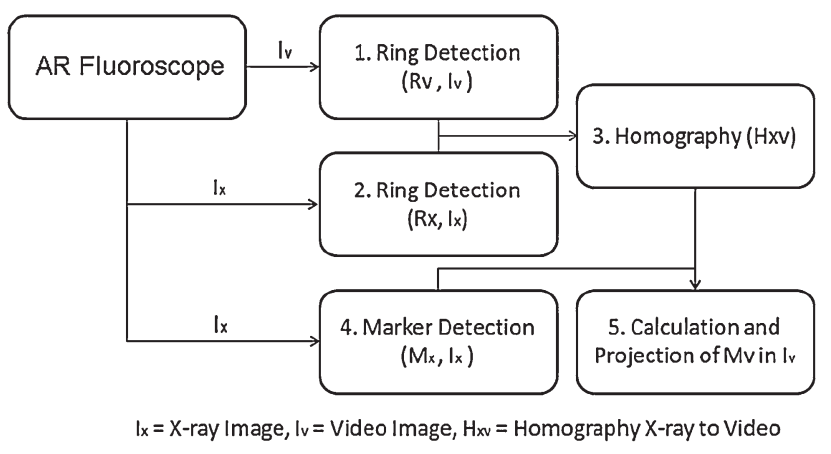

Fig. 4 Video-guided calibration workflow. Using the OpenCV segmentation tools, the phantom rings and markers visible in both the $\mathrm{X}$-ray and video images need to be processed. The correct homography is attained once these are aligned via video guidance

the direct linear transformation (DLT) can be employed to compute $\boldsymbol{H}$ with at least 4 corresponding points in the X-ray and video images [25]. The complete calibration workflow is described below.

\section{Existing calibration workflow}

WF1: Position randomly the five metallic rings on the top layer of the biplane phantom and acquire one X-ray image.

WF2: Move the rings toward their destination according to the X-ray image on the screen. Acquire an X-ray image for confirmation.

WF3: If any pair of 5 rings and markers is not isocentric, repeat WF2 until quasi-perfect superposition. Acquire a final X-ray for confirmation.

\section{Video-guided calibration}

Using the existing calibration method, once the first X-ray image is acquired, the user has to intuitively maneuver the five rings on the top plane to match the fixed markers on the lower plane with the guidance of only X-ray images. Hence, both the speed and the number of subsequent X-ray image acquisitions for ring/marker iso-centricity confirmation depend partly on the experience and partly on the luck of the user. In this light, we propose a new method exploiting video-based guidance, which marks the target destination of five movable rings in the video and provides real-time visual ring detection. This method consequently allows for calibration speed-up, reduction in X-ray image acquisitions, and real-time confirmation of overlap accuracy (Fig. 4).

Novel calibration workflow

As it is shown in Fig. 4, any point $\mathbf{I}_{\mathbf{x}} \in \mathbf{I m g}_{\mathbf{x r a y}}$ of plane $\pi$ can be transferred to $\mathbf{I}_{\mathbf{v}} \in \mathbf{I m g}_{\mathbf{v i d e o}}$ and vice versa once the homography $\boldsymbol{H}$ is obtained. From this idea and the isocentric property of the rings and markers in the final X-ray image, the position of the rings in the final X-ray image can be determined if all the markers can be detected. If this is the case, the homography of the upper plane of the calibration phantom can be used to map the position of rings to their position in the video image. First, the homography $\left(\mathbf{H}_{\mathbf{x v}}\right)$ of the top plane can easily be calculated through the center positions of the rings in both X-ray $\left(\mathbf{R}_{\mathbf{x}}\right)$ and video images $\left(\mathbf{R}_{\mathbf{v}}\right.$ ) (Fig. 5-left, center) such that any point on top plane of the calibration phantom, in X-ray image, can be transferred to the video image:

$I_{v}=H_{x v} * I_{x}$

Second, the destinations of the rings in the X-ray image $\left(\mathbf{M}_{\mathbf{x}}\right)$ are the centers of markers $\left(\mathbf{P}_{\mathbf{x y}}\right)$ because of the isocentric property of the biplane phantom (Fig. 5-right). The points $\left(\mathbf{P}_{\mathbf{x y}}\right)$ in the X-ray image $\left(\mathbf{M}_{\mathbf{x}}\right)$ are then transferred to the video image $\left(\mathbf{M}_{\mathbf{v}}\right)$ by the homography $\left(\mathbf{H}_{\mathbf{x v}}\right)$. The points are finally plotted on the graphical user interface (GUI) of the existing AR fluoroscopy system. This position will hold true if the phantom is not moved. Lastly, the user will manually guide the rings to position using the GUI and video augmentation. This in turn signifies that the rings have been correctly positioned in the X-ray image. After positioning the rings accurately using video guidance, an X-ray image is taken to confirm the alignment—but is not mandatory.

Video-guided calibration algorithm

The position of rings and markers in either X-ray or video images is detected using libraries provided by OpenCV [32]. The algorithmic sequence is presented in (Fig. 4). Our new algorithm is implemented as an additional feature to the existing GUI framework of the AR fluoroscopy system. Video augmentation of the target position of rings is developed using OpenGL libraries [33]. The circular rings are colored black to enhance the contrast, thus simplifying the detection in the video images. Since the internal parameters (i.e., focus, brightness, etc.) of the attached camera to the C-arm do not change, the algorithm parameter values listed below were determined empirically and were considered robust for our system (all parameter units and thresholds in pixels).

\section{Step 1: Detection of Rings in Video Image}

Ring centers in the video image have to be detected. To simplify this process, a contour-detection algorithm is used. The contour-detection algorithm [34] uses a binary image obtained by thresholding (threshold $=10$ ) the grayscale video image over a suitable intensity value. First, all detected contours are filtered based on their size (i.e., the sizes of the ring are known a priori). Then, those 

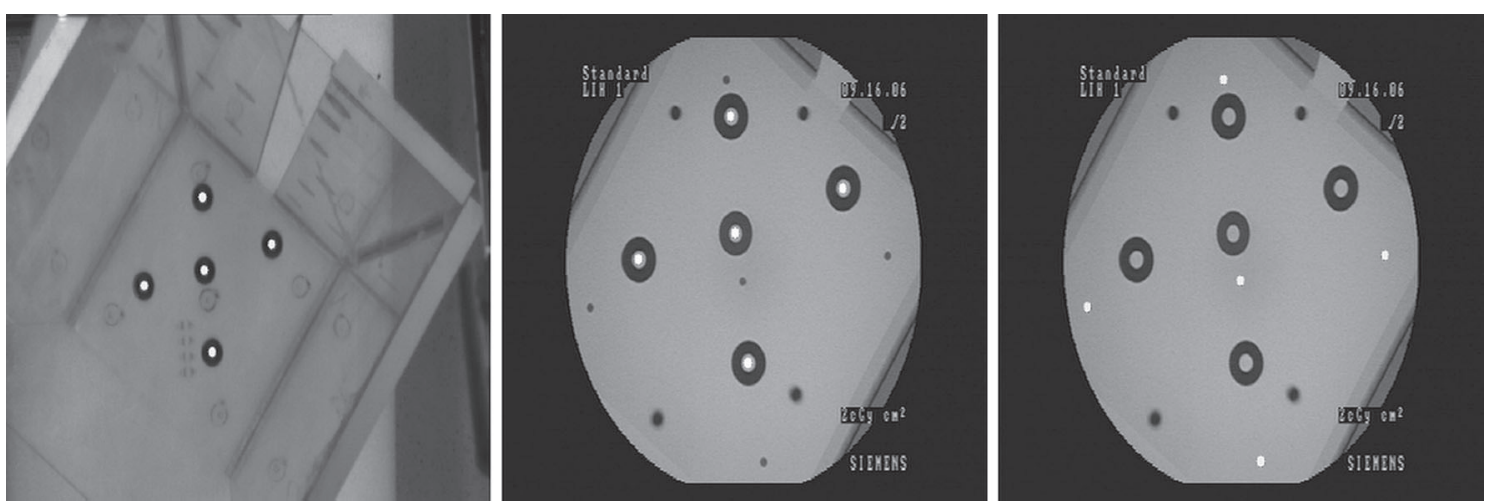

Fig. 5 X-ray and video images depicting the segmentation of rings and markers. Left centers of rings in the video image (white dots). Center centers of rings in the X-ray image (white dots) Right centers of markers in the X-ray image (white dots)

contours are processed with an ellipse fitting method to identify false positives and remove them from the true rings. Lastly, the approximate centers of the circular rings $\left(R_{v}\right)$ are found. Figure 5-left shows a white circle drawn inside the detected rings representing the center points. The parameter values for the selection criteria in the contour identification follow: area $>150$ pixels, eccentricity of contour $<1.5$, and ellipse fitting accuracy $<3$ ).

Step 2: Detection of Rings in the X-ray Image

Using a similar method, but with a different threshold value (Threshold $=80$ ), the center of rings in Xray images $\left(R_{x}\right)$ is identified. Figure 5 -center shows the detected points.

\section{Step 3: Detection of Markers in the X-ray Image}

Using the same algorithm as in Step 1, marker positions in X-ray images are identified. Markers are detected by modifying the filtering process of contour detection based on the fact that the marker sizes are considerably smaller than the circular rings. We obtain a new set of points which are displayed in Fig. 5-right.

Step 4: Calculation of Homography

In OpenCV, the origin of the image is defined as the top left corner of the image. The $\mathrm{X}$-axis of the coordinates is defined by the top left and top right corners and the direction points from the top left corner to the top right corner. The Y-axis is defined in the same way by the vector that points from top left corner to bottom left corner together with the origin. The contours detection is performed following the top-down and left-right routine. Thus, when all five rings are detected in both X-ray and video images, the corresponding points are determined by the order of the ring detection. Using these point correspondences, the homography $\mathbf{H}_{\mathbf{x v}}$ in (2) is calculated.

Step 5: Ring positions for video-based augmentation Using the homography obtained in Step 4, points inside $\mathbf{M}_{\mathbf{x}}$ are transferred to video image $\mathbf{M}_{\mathbf{v}}$. Points inside $\mathbf{M}_{\mathbf{v}}$ are augmented with a ' + ' sign in the GUI. There is a circle drawn surrounding the ' + ' to help the user manually position the rings. Note: the algorithm is initialized when all five markers are detected in the X-ray image and all five rings are detected in both $\mathrm{X}$-ray and video images.

The graphical user interface (GUI)

The aim of our experiments is to help the user move the rings on the top layer, radiation free, to the position where the fixed $\mathrm{X}$-ray visible markers are isocentric. The final position of the five rings in video images can be calculated as stated above. The target points for the user are defined by the intersection of two orthogonal white lines ('+') surrounded by red circles. The ring centers are detected in real time and defined by similar white crosses and surrounded by green circles in order to distinguish them from the target points (Fig. 6a). Each target point is assigned a number (1-5) that is also displayed in the GUI. Each moving ring will also have the same number as the target point when it is moved into the neighborhood $<$ 5 pixels away. When any one of the rings is moved into the neighborhood of a marker, the distance between the center of the ring and the corresponding point, which is defined as residual non-isocentric error, is displayed to the user in real time. The reprojection error in the X-ray image calculated by the homography is also displayed in real time (Fig. 6b). The calibration is concluded once the residual non-isocentric error of all the five pairs of rings and target positions is at most 1 pixel (Fig. 6c). A value of 1 pixel equals the required precision needed to translate the AR fluoroscope within the operating room setting [24].

The video-guided workflow sequential steps follow.

Video-guided calibration workflow

WF1: Position the five metallic rings at the center of the top layer of the biplane phantom. Acquire one X-ray image.

WF2: Start the video-guidance calibration program and GUI. Overlay rings accordingly as in Fig. 6c. 


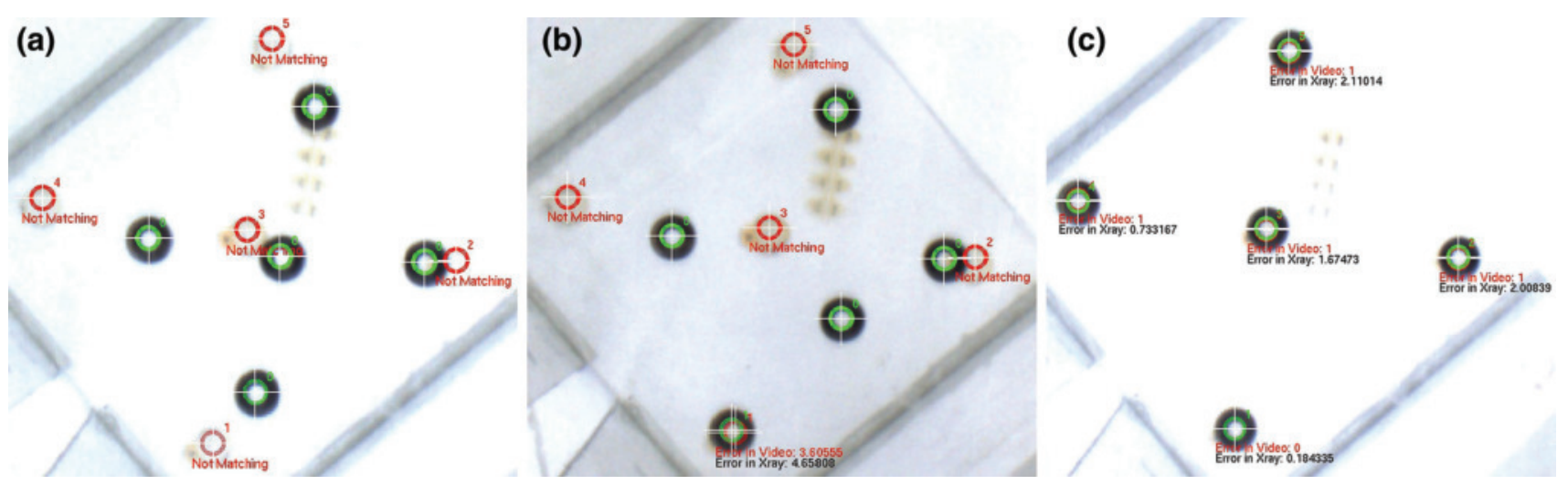

Fig. 6 The GUI showing the target positions of the five rings in red alongside the five metallic rings drawn in green. All detections are performed in real time. Each circle representing the target position and each ring have a number of its own shown (\#1-\#5) in its north east direction. Left "not matching" is shown under each of the red circles representing the target positions. Center if the ring is moved to the place where the distance between the ring and the target position is smaller than 5 pixels, the number of the green circle is set as the same as the number of the red circle. Both video overlay and reprojection error in $\mathrm{X}$-ray (i.e., pixels) are shown in red and black, respectively. Right when the distance of each of the five rings in the video image is no more than 1pixel, the calibration process is considered completed
Fig. 7 a The total time required to complete the first phase of the calibration of the AR fluoroscope. $\mathbf{b}$ The total number of X-ray shots required to complete the first phase of the calibration of the AR fluoroscope (a)

Total time in minutes required for calibration

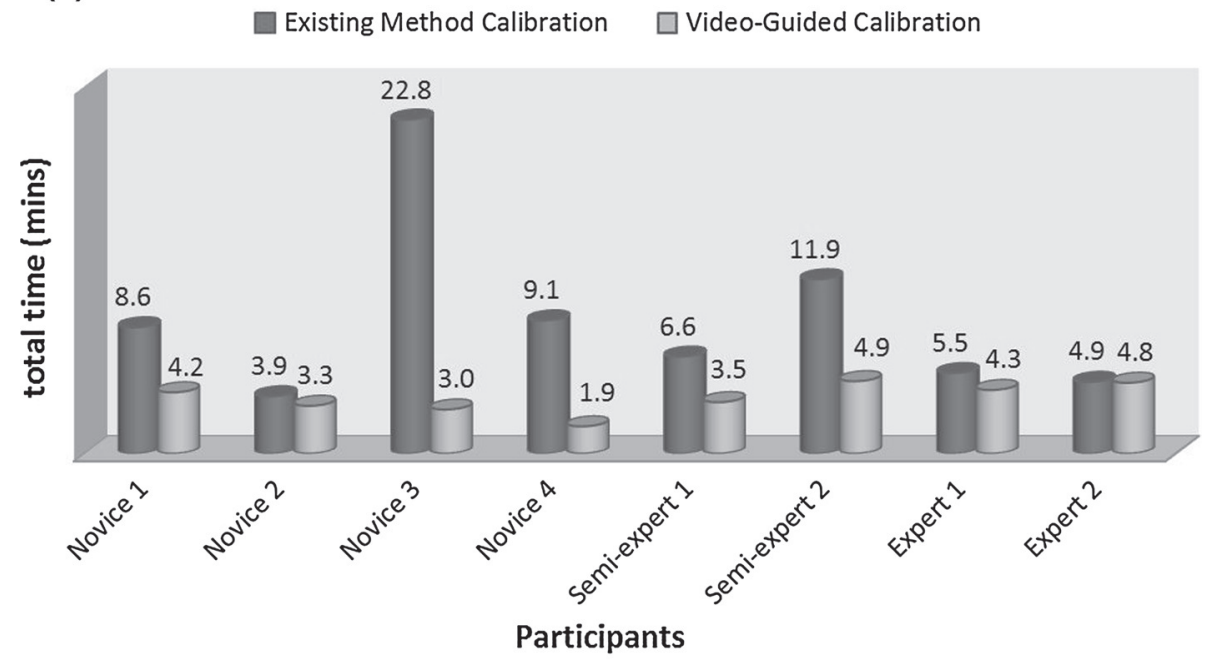

(b)

\section{Number of $X$-ray shots required for calibration}

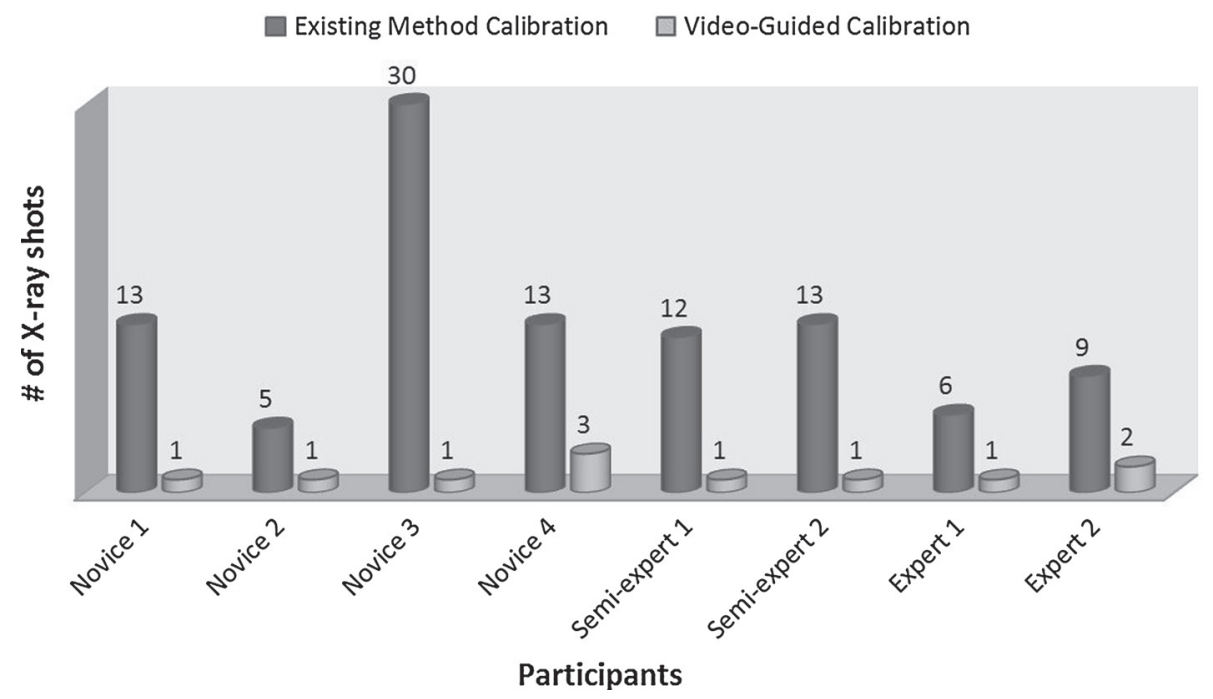


WF3: When distance between each ring and its target destination in video is $<1$ pixel, calibration is complete.

\section{Optional steps}

WF4: User has the option of acquiring an additional $\mathrm{X}$-ray image to confirm superposition. This step is not mandatory.

WF5: If the user wants improved precision $(\sim 0$ pixel error) - move ring(s) accordingly and take additional $\mathrm{X}$-ray image (s) until satisfied.

\section{Experimentation protocol}

As part of a preclinical evaluation, eight participants were asked to perform each calibration once. In total, four participants never performed the existing calibration previously (i.e., novice group), two participants performed the existing calibration once previously (i.e., semi-expert group), and two participants performed the traditional calibration multiple times previously (i.e., expert group). The participants did not undergo training prior to performing the new videoguided calibration technique.

The metrics that were considered during the evaluation were: (i) total number of $\mathrm{X}$-ray image acquisitions to complete calibration and (ii) total amount of time required to complete the two calibrations methods individually. Lastly, a one-way ANOVA analysis was performed to assess the statistical significance $(p<0.05)$ between the calibration methods.

\section{Results}

Figure 7a, b depicts the experimental results of each participant when completing the first phase of the calibration. Using our proposed video-guided method, the time required to complete the calibration was $4.57 \pm 0.65$ min compared to 9.14 $\pm 6.08 \mathrm{~min}$ using the existing method. Similarly, the total number of X-ray acquisitions required by the participants using video-guidance was $1.38 \pm 0.74$ compared to $12.63 \pm$ 7.73 using the existing calibration. The video-guided process showed statistical significance in time $(p=0.026, F=$ 6.16) and number of X-ray shots ( $p=0.001, F=16.8)$ compared to the existing calibration process. The isocentric pixel error using the traditional calibration was 1.33 pixels compared to 1.40 pixels using video guidance.

Next, we present results based on the individual expertise of the participants. For the novice group, an average of $4.54 \pm 0.83 \mathrm{~min}$ and $1.50 \pm 1.00 \mathrm{X}$-rays was achieved using video-guidance compared to $11.08 \pm 8.12 \mathrm{~min}$ and 15.25 $\pm 10.53 \mathrm{X}$-rays for the existing calibration method. For the

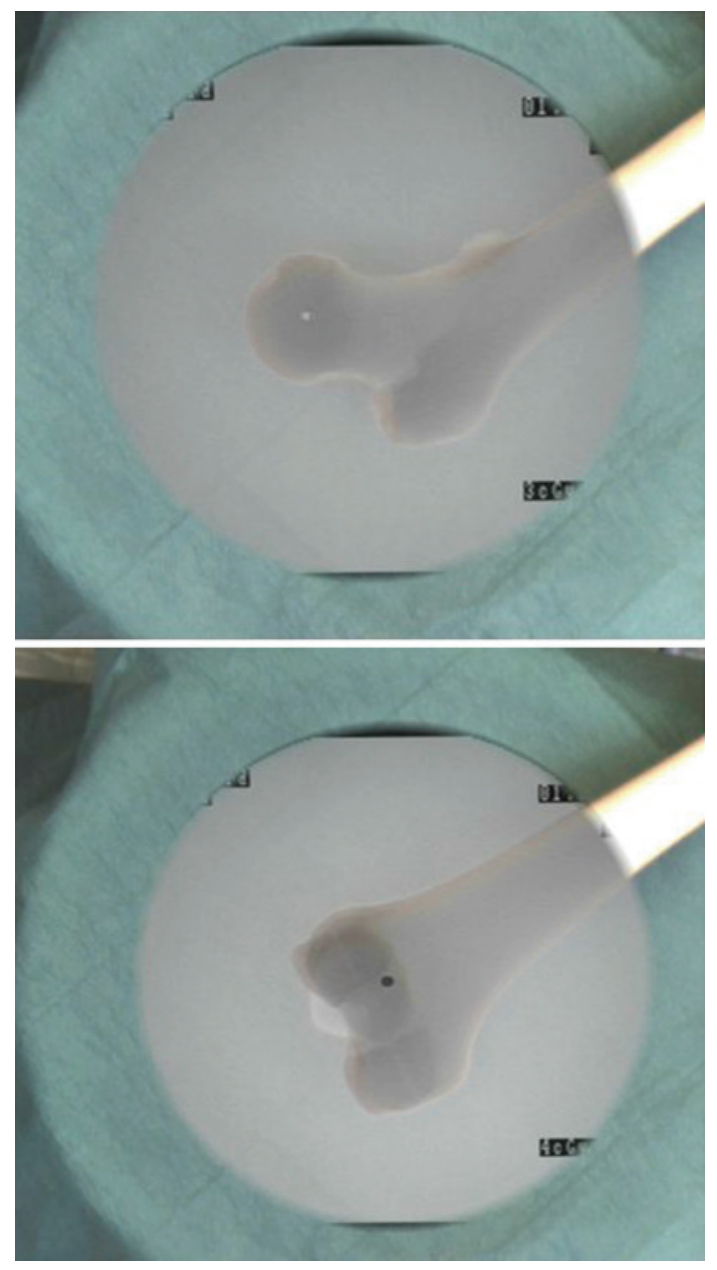

Fig. 8 Dry bone phantoms of the hip and knee, with the AR fluoroscopic overlay after video-based calibration

semi-expert group, an average of $4.63 \pm 0.42 \mathrm{~min}$ and $1.0 \pm$ $0.0 \mathrm{X}$-rays was achieved using video-guidance compared to $9.24 \pm 3.73 \mathrm{~min}$ and $12.50 \pm 0.71 \mathrm{X}$-rays for the existing calibration method. Lastly, for the expert group, an average of $4.58 \pm 0.35 \mathrm{~min}$ and $1.50 \pm 0.71 \mathrm{X}$-rays was achieved using video-guidance compared to $5.18 \pm 0.42 \mathrm{~min}$ and 7.50 $\pm 2.12 \mathrm{X}$-rays for the existing calibration method.

\section{Discussion and conclusions}

In this paper, we have proposed an elegant solution to calibrate accurately an augmented reality fluoroscope that is currently used to assist navigation and completion of orthopedic and trauma procedures. We exploit the video feedback of the AR fluoroscopy device by detecting rings in real time which assists the user in aligning accurately both the Xray and video camera centers. The GUI offers a real-time visual of the non-isocentric pixel errors between movable circular rings and their target destinations. During the exper- 
iments, we asked all participants to achieve at most 1 pixel error for each of the 5 rings. In the ideal scenario, a quasiperfect overlay error in video can be achieved (i.e., $\sim 0$ pixels). The lower non-isocentric error in the video image yields a lower non-isocentric error in the X-ray image. From this, we believe it is better for any user to be patient and move the rings as close as possible to their targets in the video image. Via training, we anticipate a quicker video-guided calibration.

Ideally, this calibration process can be completed by one $\mathrm{X}$-ray shot using our proposed video-guidance technique. Until now, the existing AR fluoroscopy system calibration was time consuming and radiation excessive. This is a problem if the AR fluoroscope was to become miscalibrated prior to or during surgery (i.e., due to mirrors being unintentionally moved, etc.). From the evaluation, our video augmented calibration reduces on average the number of X-ray images required by $89 \%$ and decreases the total time required by $59 \%$ for calibration confirmation and image overlay accuracy (Fig. 8). In conclusion, we showed that the existing calibration process of the AR fluoroscope is complex however by exploiting the video camera, we can assist any user by video guidance to achieve desired overlay results in less time with minimal exposure to X-ray.

Conflict of interst Xin Chen, Hemal Naik, Lejing Wang, Nassir Navab, Pascal Fallavollita have no conflict of interest.

\section{References}

1. Paul P, Fleig O, Jannin P (2005) Augmented virtuality based on stereoscopic reconstruction in multimodal image-guided neurosurgery: methods and performance evaluation. IEEE Trans Med Imaging 24(11):1500-1511

2. Birkfellner W, Figl M, Huber K, Watzinger F, Wanschitz F, Hummel J, Hanel R, Greimel W, Homolka P, Ewers R, Bergmann H (2002) A head-mounted operating binocular for augmented reality visualization in medicine-design and initial evaluation. IEEE Trans Med Imaging 21(8):991-997

3. Grimson WEL, Lozano-Perez T, Wells WM III, Ettinger GJ, White SJ, Kikinis R (1996) An automatic registration method for frameless stereotaxy, image guided surgery, and enhanced reality visualization. IEEE Trans Med Imaging 15(2):129-140

4. Bajura M, Fuchs H, Ohbuchi R (1992) Merging virtual objects with the real world: seeing ultrasound imagery within the patient. In: Proceedings of the 19th annual conference on computer graphics and interactive techniques. ACM Press, pp 203-210

5. State A, Chen DT, Tector C, Brandt A, Chen H, Ohbuchi R, Bajura M, Fuchs H (1994) Case study: observing a volume rendered fetus within a pregnant patient. In: Proceedings of the conference on visualization '94. IEEE Computer Society Press, pp 364-368

6. Sauer F, Khamene A, Bascle B, Rubino GJ (2001) A head-mounted display system for augmented reality image guidance: towards clinical evaluation for imri-guided neurosurgery. In: Proceeings of the international conference on medical image computing and computer assisted intervention (MICCAI). Springer, London, pp 707716
7. Sauer F, Schoepf UJ, Khamene A, Vogt S, Das M, Silverman SG (2003) Augmented reality system for ct-guided interventions: system description and initial phantom trials. In: Medical imaging: visualization, image-guided procedures, and display

8. Blackwell M, Nikou C, DiGioia AM, Kanade T (2000) An image overlay system for medical data visualization. Med Image Anal 4(1):67-72

9. Sielhorst T, Feuerstein M, Navab N (2008) Advanced medical displays: a literature review of augmented reality. IEEE/OSA J Disp Technol. Special issue on Medical Displays, 4(4):451-467

10. Stetten G, Cois A, Chang W, Shelton D, Tamburo R, Castellucci J, von Ramm O (2005) C-mode real-time tomographic reflection for a matrix array ultrasound sonic flashlight. Acad Radiol 12: 535-543

11. Fichtinger G, Deguet A, Masamune K, Balogh E, Fischer GS, Mathieu H, Taylor RH, Zinreich SJ, Fayad LM (2005) Image overlay guidance for needle insertion in CT scanner. IEEE Trans BioMed Eng 52:1415-1424

12. Fischer GS, Deguet A, Csoma C, Taylor RH, Fayad L, Carrino JA, Zinreich SJ, Fichtinger G (2007) MRI image overlay: application to arthrography needle insertion. Comput Aided Surg Off J Int Soc Comput Aided Surg 12:2-14

13. Feuerstein M, Mussack T, Heining SM, Navab N (2008) Intraoperative laparoscope augmentation for port placement and resection planning in minimally invasive liver resection. IEEE Trans Med Imaging 27:355-369

14. Wendler T, Feuerstein M, Traub J, Lasser T, Vogel J, Daghighian F, Ziegler SI, Navab N (2007) Real-time fusion of ultrasound and gamma probe for navigated localization of liver metastases. In: International conference on medical image computing and computer-assisted intervention MICCAI 10, pp 252-260

15. Moult E et al (2011) Automatic C-arm pose estimation via 2D/3D hybrid registration of a radiographic fiducial. In: SPIE medical imaging. p 79642S

16. Fallavollita $\mathrm{P}$ et al (2010) C-arm pose estimation in prostate brachytherapy by registration to ultrasound. In: MICCAI (3). LNCS, Springer, pp 311-318

17. Navab N, Mitschke M, Schütz O (1999) Camera-augmented mobile C-arm (CAMC) application: 3D reconstruction using a low-cost mobile C-arm. In: International conference on medical image computing and computer-assisted intervention, Lecture notes in computer science, vol 1679/1999, pp 688-697

18. Navab N, Heining SM, Traub J (2010) Camera augmented mobile C-arm (CAMC): calibration, accuracy study, and clinical applications. IEEE Trans Med Imaging 29(7):1412-1423

19. Wang L, Traub J, Weidert S, Heining SM, Euler E, Navab N (2010) Parallax-free intraoperative X-ray image stitching. Med Image Anal 14(5):674-686

20. Navab N, Wiesner S, Benhimane S, Euler E, Heining SM (2006) Visual servoing for intraoperative positioning and repositioning of mobile C-arms. In: International conference on medical image computing and computer-assisted intervention, vol 19, no 1, pp 551-560

21. Wang L, Landes J, Weidert S et al (2010) First animal cadaver study for interlocking of intramedullary nails under camera augmented mobile C-arm. A surgical workflow based preclinical evaluation. In: IPCAI'10 Proceedings of the first international conference on information processing in computer-assisted interventions, pp 5666

22. Diotte B, Fallavollita P, Wang L, Weidert S, Thaller P, Euler E, Navab N (2012) Radiation-free drill guidance in interlocking of intramedullary nails. In: 15 th international conference on medical image computing and computer-assisted intervention, vol 7510, pp $18-25$

23. Londei R, Esposito M, Diotte B, Weidert S, Euler E, Thaller P, Navab N, Fallavollita P (2014) The 'augmented' circles: a video- 
guided solution for the down-the-beam positioning of IM nail holes. In: Information processing in computer-assisted interventions (IPCAI) (in press)

24. Wang L, Fallavollita P, Zou R, Chen X, Weidert S, Navab N (2012 May) Closed-form inverse kinematics for interventional C-arm Xray imaging with six degrees of freedom: modeling and application. IEEE Trans Med Imaging 31(5):1086-1099

25. Chen X, Wang L, Fallavollita P, Navab N (2013) Precise X-ray and video overlay for augmented reality fluoroscopy. Int J Comput Assist Radiol Surg 8(1):29-38

26. Chen X, Wang L, Fallavollita P, Navab N (2012) Error analysis of the X-ray projection geometry of camera-augmented mobile Carm. In: SPIE medical imaging. International Society for Optics and Photonics, pp 83160P-83160P

27. Pati S, Erat O, Wang L, Weidert S, Euler E, Navab N, Fallavollita P (2013) Accurate pose estimation using single marker single camera calibration system. In: SPIE medical imaging. International Society for Optics and Photonics, pp 867126-867126

28. Wang L, Fallavollita P, Brand A, Erat O, Weidert S, Thaller P-H, Euler E, Navab N (2012) Intra-op measurement of the mechanical axis deviation: an evaluation study on 19 human cadaver legs. In: Medical image computing and computer-assisted interventionMICCAI. Springer, Berlin, pp 609-616
29. Erat O, Pauly O, Weidert S, Thaller P, Euler E, Mutschler W, Navab N, Fallavollita P (2013) How a surgeon becomes superman by visualization of intelligently fused multi-modalities. In: SPIE medical imaging. International Society for Optics and Photonics, pp 86710L-86710L

30. Pauly O, Katouzian A, Eslami A, Fallavollita P, Navab N (2012) Supervised classification for customized intraoperative augmented reality visualization. In: IEEE international symposium on mixed and augmented reality (ISMAR), pp 311-312

31. Pauly O, Diotte B, Habert S, Weidert S, Euler E, Fallavollita $P$, Navab N (2014) Visualization inside the operating room: "learning" what the surgeon wants to see. In: Information processing in computer-assisted interventions (IPCAI) (in press)

32. Bradski G (2000) The OpenCV library. Dr Dobb's J 25(11):120126

33. Wright R et al (2010) OpenGL SuperBible: comprehensive tutorial and reference. Pearson Education, Upper Saddle River

34. Suzuki S, Abe K (1985) Topological structural analysis of digital binary images by border following. CVGIP 30(1):32-46 